\author{
DMYTRYSHYN M. ${ }^{1}$, LOPUSHANSKY O. ${ }^{2}$
}

\title{
SPECTRAL APPROXIMATIONS OF STRONGLY DEGENERATE ELLIPTIC DIFFERENTIAL OPERATORS
}

\begin{abstract}
We establish analytical estimates of spectral approximations errors for strongly degenerate elliptic differential operators in the Lebesgue space $L_{q}(\Omega)$ on a bounded domain $\Omega$. Elliptic operators have coefficients with strong degeneration near boundary. Their spectrum consists of isolated eigenvalues of finite multiplicity and the linear span of the associated eigenvectors is dense in $L_{q}(\Omega)$. The received results are based on an appropriate generalization of Bernstein-Jackson inequalities with explicitly calculated constants for quasi-normalized Besov-type approximation spaces which are associated with the given elliptic operator. The approximation spaces are determined by the functional $E(t, u)$, which characterizes the shortest distance from an arbitrary function $u \in L_{q}(\Omega)$ to the closed linear span of spectral subspaces of the given operator, corresponding to the eigenvalues such that not larger than fixed $t>0$. Such linear span of spectral subspaces coincides with the subspace of entire analytic functions of exponential type not larger than $t>0$. The approximation functional $E(t, u)$ in our cases plays a similar role as the modulus of smoothness in the functions theory.

Key words and phrases: elliptic operators, spectral approximations.
\end{abstract}

\footnotetext{
${ }^{1}$ Vasyl Stefanyk Precarpathian National University, 57 Shevchenka str., 76018, Ivano-Frankivsk, Ukraine

${ }^{2}$ University of Rzeszow, 1 Prof. St. Pigonia str., 35-310, Rzeszow, Poland

E-mail: marian.dmytryshyn@pnu.edu.ua (Dmytryshyn M.), ovlopusz@ur.edu.pl (Lopushansky O.)
}

\section{INTRODUCTION}

We investigate the problem of best approximations in the Lebesgue space $L_{q}(\Omega)$ on a bounded domain $\Omega \subset \mathbb{R}^{n}$ by using spectral subspaces $\mathcal{R}(A)$ of a strongly degenerate elliptic differential operator $A$. Our aims is to prove the inverse and direct theorems that give precise estimates of approximation errors and which are connected with appropriate estimations by Bernstein-Jackson type inequalities.

For this purpose we use the best approximation functional $E\left(t, u ; \mathcal{R}(A), L_{q}(\Omega)\right)$ which characterizes a shortest distance from an arbitrary function $u \in L_{q}(\Omega)$ to the closed linear span $\mathcal{R}^{t}(A)$ of all spectral subspaces $\mathcal{R}_{\lambda_{j}}(A)$ of the given operator $A$, corresponding to the eigenvalues $\lambda_{j}$ such that $\left|\lambda_{j}\right|<t$ with a fixed $t>0$.

This best approximation problem we solve by finding exact values of constants in the Bernstein-Jackson inequalities. Namely, we establish the Bernstein-Jackson inequalities with explicitly calculated constants, using the suitable generalization of $\operatorname{Besov}^{\prime}$ space $\mathcal{B}_{r}^{s}\left(A, L_{q}(\Omega)\right)$, determined by a given operator $A$ and an appropriate functional $E\left(t, u ; \mathcal{R}(A), L_{q}(\Omega)\right)$.

It is essentially to note that the approximation functional $E\left(t, u ; \mathcal{R}(A), L_{q}(\Omega)\right)$ in these inequalities plays a similar role as the modulus of smoothness in the functions theory. Earlier applications of smoothness modulus to approximation problems can be found in [5-7].

In this paper we continue the research started in $[3,4]$.

$\mathrm{Y} \Delta \mathrm{K} 517.956 .2$

2010 Mathematics Subject Classification:35J30, 47A58.

(C) Dmytryshyn M., Lopushansky O., 2019 


\section{STRONGLY DEGENERATE ELLIPTIC DIFFERENTIAL OPERATORS}

We shall follow the treatment given in [8, Sec. 6.2.1]. Let $\Omega \subset \mathbb{R}^{n}$ be an open bounded set with the infinitely smooth boundary $\partial \Omega$. As usual, $C^{\infty}(\Omega)$ denotes the space of all infinitely differentiable complex-valued functions defined on $\Omega$. Suppose that $\rho(x) \in C^{\infty}(\Omega)$ is a positive function such that:

(i) for all multi-indices $\alpha=\left(\alpha_{1}, \ldots, \alpha_{n}\right) \in \mathbb{N}^{n},|\alpha|=\alpha_{1}+\ldots+\alpha_{n}$ there exist positive numbers $c_{\alpha}$ such that

$$
\left|D^{\alpha} \rho(x)\right| \leq c_{\alpha} \rho^{1+|\alpha|}(x) \text { for all } x \in \Omega
$$

(ii) for any positive number $K$ there exist numbers $\varepsilon_{K}>0$ and $r_{K}>0$ such that $\rho(x)>K$, if $d(x) \leq \varepsilon_{K}$ or $|x| \geq r_{K}, x \in \Omega$ (here, $d(x)$ is the distance to the boundary $\partial \Omega$ ).

In what follows, $S_{\rho(x)}(\Omega)$ denotes the locally convex space

$$
S_{\rho(x)}(\Omega)=\left\{u: u \in C^{\infty}(\Omega),\|u\|_{l, \alpha}=\sup _{x \in \Omega} \rho^{l}(x)\left|D^{\alpha} u(x)\right|<\infty \text { for all } \alpha \text { and } l \in \mathbb{N}_{0}\right\} .
$$

Let $m \in \mathbb{N}, \mu, \tau \in \mathbb{R}$ and $\tau>\mu+2 m$. We put

$$
\aleph_{l}=\frac{1}{2 m}(\tau(2 m-l)+\mu l), l=0,1, \ldots, 2 m,
$$

and consider the differential elliptic operator

$$
A u=\sum_{l=0}^{m} \sum_{|\alpha|=2 l} \rho^{\aleph_{2 l}}(x) b_{\alpha}(x) D^{\alpha} u+\sum_{|\beta|<2 m} a_{\beta}(x) D^{\beta} u,
$$

where $b_{\alpha}(x) \in C^{\infty}(\Omega)(|\alpha|=2 l, l=0,1, \ldots, m)$ are real functions, all derivatives of which (inclusively the functions themselves) are bounded in $\Omega$. In sequel we assume that there exists a positive number $C$ such that for all $\xi \in \mathbb{R}^{n}$ and all $x \in \Omega$

$$
\begin{gathered}
(-1)^{m} \sum_{|\alpha|=2 m} b_{\alpha}(x) \xi^{\alpha} \geq C|\xi|^{2 m}, \quad b_{(0, \ldots, 0)}(x) \geq C, \\
(-1)^{l} \sum_{|\alpha|=2 l} b_{\alpha}(x) \xi^{\alpha} \geq 0, \quad l=1, \ldots, m-1 .
\end{gathered}
$$

Moreover, let $a_{\beta}(x) \in C^{\infty}(\Omega)(0 \leq|\beta|<2 m)$ and there exists a positive number $\delta>0$ such that $D^{\gamma} a_{\beta}(\xi)=\mathrm{O}\left(\rho^{\aleph}|\beta|+|\gamma|-\delta\right)$ for $0 \leq|\beta|<2 m$ and for all multi-indices $\gamma$.

Let $1<q<\infty, \tau \geq \mu+s q, s \in \mathbb{N}_{0}$ and $\tau, \mu \in \mathbb{R}$. Consider the weighted Sobolev space $W_{q}^{s}\left(\Omega ; \rho^{\mu} ; \rho^{\tau}\right)$ endowed with the norm (see $[8$, Thm 3.2.4/2])

$$
\|u\|_{W_{q}^{s}\left(\Omega ; \rho^{\mu} ; \rho^{\tau}\right)}=\left[\int_{\Omega}\left(\sum_{|\alpha|=s} \rho^{\mu}(x)\left|D^{\alpha} u(x)\right|^{q}+\rho^{\tau}(x)|u(x)|^{q}\right) d x\right]^{\frac{1}{q}} .
$$

Let $\tau>0,1<q<\infty$ and $\rho^{-a}(x) \in L_{1}(\Omega)$ for an appropriate number $a \geq 0$. Then $A$ given by (1) with the domain $\mathfrak{D}(A)=W_{q}^{2 m}\left(\Omega ; \rho^{q \mu} ; \rho^{q \tau}\right)$ is the closed operator in $L_{q}(\Omega)$ (see [8, Thm 6.6.2]). The spectrum of $A$ consists of isolated eigenvalues $\left\{\lambda_{j} \in \mathbb{C}: j \in \mathbb{N}\right\}$ of finite algebraic 
multiplicity and its eigenvectors belongs to $S_{\rho(x)}(\Omega)$, as well as, its linear span is dense in $S_{\rho(x)}(\Omega)$ and, as a consequence, it is dense in $L_{q}(\Omega)$.

Let $\mathcal{R}_{\lambda_{j}}(A)=\left\{u \in \mathfrak{D}^{\infty}(A)=\bigcap_{k \in \mathbb{N}} \mathfrak{D}^{k}(A):\left(\lambda_{j}-A\right)^{r_{j}} u=0\right\}$ be the spectral subspace, corresponding to the eigenvalue $\lambda_{j}$ of multiplicity $r_{j}$. Denote by $\mathcal{R}^{v}(A)$ the complex linear span in $L_{q}(\Omega)$ of all spectral subspaces $\mathcal{R}_{\lambda_{j}}(A)$ such that $\left|\lambda_{j}\right|<v$. Following to [4], let $\mathcal{R}(A):=\bigcup_{v>0} \mathcal{R}^{v}(A)$ be endowed with the quasi-norm

$$
|u|_{\mathcal{R}(A)}=\|u\|_{L_{q}(\Omega)}+\inf \left\{v>0: u \in \mathcal{R}^{v}(A)\right\} .
$$

\section{ANALYTICAL ESTIMATES OF SPECTRAL APPROXIMATIONS}

Let us consider the subspace of all exponential type vectors $\mathcal{E}(A)$ of the elliptic operator $A$ as the union $\bigcup_{v>0} \mathcal{E}^{v}(A)$ which is endowed with the quasi-norm

$$
|u|_{\mathcal{E}(A)}=\|u\|_{L_{q}(\Omega)}+\inf \left\{v>0: u \in \mathcal{E}^{v}(A)\right\},
$$

where for any $v>0$ the subspace $\mathcal{E}^{v}(A)=\left\{u \in \mathcal{E}(A):\|u\|_{\mathcal{E}^{v}(A)}<\infty\right\}$ is endowed with the norm $\|u\|_{\mathcal{E}^{v}(A)}=\sum_{k \in \mathbb{N}_{0}}\left\|(A / v)^{k} u\right\|_{L_{q}(\Omega)}($ see $[3,4])$.

Let $0<s<\infty$ and $0<r \leq \infty$ or $0 \leq s<\infty$ and $r=\infty$. To investigate spectral approximation errors, we consider the appropriate Besov spaces

$$
\mathcal{B}_{r}^{s}\left(A, L_{q}(\Omega)\right)=\left\{u \in L_{q}(\Omega):|u|_{\mathcal{B}_{r}^{s}\left(A, L_{q}(\Omega)\right)}<\infty\right\}
$$

associated with the given operator $A$ on the space $L_{q}(\Omega)$, which is endowed with the norm

$$
|u|_{\mathcal{B}_{r}^{s}\left(A, L_{q}(\Omega)\right)}= \begin{cases}\left(\int_{0}^{\infty}\left[t^{s} E\left(t, u ; \mathcal{E}(A), L_{q}(\Omega)\right)\right]^{r} \frac{d t}{t}\right)^{1 / r}, & 0<r<\infty, \\ \sup _{t>0} t^{s} E\left(t, u ; \mathcal{E}(A), L_{q}(\Omega)\right), & r=\infty,\end{cases}
$$

where $E\left(t, u ; \mathcal{E}(A), L_{q}(\Omega)\right)=\inf \left\{\left\|u-u^{0}\right\|_{L_{q}(\Omega)}: u^{0} \in \mathcal{E}(A),\left|u^{0}\right|_{\mathcal{E}(A)}<t\right\}$ for all $u \in L_{q}(\Omega)$ and $t>0$. Denote $E\left(t, u ; \mathcal{R}(A), L_{q}(\Omega)\right)=\inf \left\{\left\|u-u^{0}\right\|_{L_{q}(\Omega)}: u^{0} \in \mathcal{R}(A),\left|u^{0}\right|_{\mathcal{R}(A)} \leq t\right\}$ for all $u \in L_{q}(\Omega)$.

Now, we consider the space $\mathcal{E}^{v}(D)=\left\{u \in C^{\infty}(\bar{\Omega}): D^{\alpha} u \in L_{q}(\Omega),|\alpha|=k \in \mathbb{N}_{0}\right\}$ endowed with the norm $\|u\|_{\mathcal{E}^{v}(D)}=\sum_{k \geq 0} \sum_{|\alpha|=k} v^{-k}\left\|D^{\alpha} u\right\|_{L_{q}(\Omega)}$. On $\mathcal{E}(D)=\bigcup_{v>0} \mathcal{E}^{v}(D)$ we define the quasi-norm $|u|_{\mathcal{E}(D)}=\|u\|_{L_{q}(\Omega)}+\inf \left\{v>0: u \in \mathcal{E}^{v}(D)\right\}$.

In [3, Thm 9] it is proved that $\mathcal{E}(D)$ coincides with the space $\mathcal{M}_{q}(\Omega)=\bigcup_{v>0} \mathcal{M}_{q}^{v}(\Omega)$ endowed with the quasi-norm

$$
|u|_{\mathcal{M}_{q}(\Omega)}=\inf _{\left.v\right|_{\Omega}=u, v \in L_{q}\left(\mathbb{R}^{n}\right)}\left\{\|v\|_{L_{q}\left(\mathbb{R}^{n}\right)}+\sup _{\zeta \in \operatorname{supp} F v}|\zeta|\right\}
$$

where supp $F v$ denotes the support of the Fourier-image $F v$ of a function $v \in L_{q}\left(\mathbb{R}^{n}\right)$ and $\mathcal{M}_{q}^{v}(\Omega)$ means the space of entire analytic functions $v(z)$ of the complex variable $z \in \mathbb{C}^{n}$ of an exponential type $v>0$ which restrictions to $\Omega$ belong to $L_{q}(\Omega)$. 
Taking into account $[1$, Sec. 7.2$]$ or $[8$, Sec. 2.5 .4$]$ and the mentioned above equality $\mathcal{E}(D)=$ $\mathcal{M}_{q}(\Omega)$, the classic Besov space $B_{q, r}^{s}(\Omega)$ over $\Omega$ can be endowed with the norm

$$
\|u\|_{B_{q, r}^{s}(\Omega)}= \begin{cases}\left(\int_{0}^{\infty}\left[t^{s} E\left(t, u ; \mathcal{E}(D), L_{q}(\Omega)\right)\right]^{r} \frac{d t}{t}\right)^{1 / r}, & 0<r<\infty, \\ \sup _{t>0} t^{s} E\left(t, u ; \mathcal{E}(D), L_{q}(\Omega)\right), & r=\infty .\end{cases}
$$

In $B_{q, r}^{s}(\Omega)$ we consider the subspace which is associated with the function $\rho(x)$,

$$
B_{q, r, \rho(x)}^{s}(\Omega)=\left\{u \in B_{q, r}^{s}(\Omega): \sup _{x \in \Omega} \rho^{l}(x)\left|D^{\alpha} u(x)\right|<\infty \text { for all } \alpha \text { and } l \in \mathbb{N}_{0}\right\} .
$$

Theorem 1. The following Bernstein-Jackson inequalities hold,

$$
\begin{aligned}
\|u\|_{B_{q, r}^{s}(\Omega)} & \leq c_{s, r}|u|_{\mathcal{R}(A)}^{s}\|u\|_{L_{q}(\Omega)}, \quad u \in \mathcal{R}(A), \\
t^{s} E\left(t, u ; \mathcal{R}(A), L_{q}(\Omega)\right) & \leq C_{s, r}\|u\|_{B_{q, r}^{s}(\Omega)}, \quad u \in B_{q, r, \rho(x)}^{s}(\Omega)
\end{aligned}
$$

with the constants $c_{s, r}=\left(r s^{-1}(s+1)^{2}\right)^{1 / r}$ and $C_{s, r}=2^{s+1}\left(r^{-1} s(s+1)^{-2}\right)^{1 / r}$ if $r<\infty$, $c_{s, \infty}=C_{s, \infty}=1$. In addition, for each $u \in B_{q, r, \rho(x)}^{s}(\Omega)$,

$$
\inf \left\{\left\|u-u^{0}\right\|_{L_{q}(\Omega)}: u^{0} \in \mathcal{R}^{v}(A)\right\} \leq v^{-s} C_{s, r}\|u\|_{B_{q, r}^{s}(\Omega)} .
$$

Proof. First, note that applying [2, Thm 2.2], we get the following equalities

$$
\mathcal{E}(A)=\mathcal{R}(A), \quad|u|_{\mathcal{E}(A)}=|u|_{\mathcal{R}(A)} \quad \text { for all } u \in \mathcal{E}(A) \text {. }
$$

Now, we show that the following linear topological isomorphism holds,

$$
\mathcal{B}_{r}^{\mathcal{S}}\left(A, L_{q}(\Omega)\right)=B_{q, r, \rho(x)}^{\mathcal{S}}(\Omega) .
$$

Using [8, Thm 6.5.2/1, Thm 3.2.4/3], we have

$$
\mathfrak{D}^{\infty}(A)=\bigcap \mathfrak{D}^{k}(A)=\bigcap W_{q}^{2 m k}\left(\Omega ; \rho^{q \mu k} ; \rho^{q \tau k}\right)=S_{\rho(x)}(\Omega),
$$

where the locally convex space $\mathfrak{D}^{\infty}(A)$ endowed with the semi-norms $\left\|A^{k} u\right\|_{L_{q}(\Omega)}$ for all $k \in \mathbb{N}_{0}$. Above, the equality also must be understood as linear topological isomorphism.

Let us prove the equality

$$
\mathcal{E}(A)=\left\{u \in \mathcal{E}(D): \sup _{x \in \Omega} \rho^{l}(x)\left|D^{\alpha} u(x)\right|<\infty \text { for all } \alpha \text { and } l \in \mathbb{N}_{0}\right\} .
$$

Since $\left\|A^{k} u\right\|_{L_{q}(\Omega)} \leq v^{k}\|u\|_{L_{q}(\Omega)} \leq v^{2 k}\left(\sum_{|\alpha|=k} v^{-k}\left\|D^{\alpha} u\right\|_{L_{q}(\Omega)}+v^{-k}\|u\|_{L_{q}(\Omega)}\right)$ for all $u \in \mathcal{E}^{v}(A)$, we get $\sum v^{-2 k}\left\|A^{k} u\right\|_{L_{q}(\Omega)} \leq \sum\left(\sum_{|\alpha|=k} v^{-k}\left\|D^{\alpha} u\right\|_{L_{q}(\Omega)}+v^{-k}\|u\|_{L_{q}(\Omega)}\right)$. Substituting $\sigma=v^{2}$ with $v>1$, we have

$$
\|u\|_{\mathcal{E}^{\sigma}(A)} \leq\|u\|_{\mathcal{E}^{v}(D)}+\frac{v\|u\|_{L_{q}(\Omega)}}{v-1} \leq\|u\|_{\mathcal{E}^{v}(D)}+\frac{v\|u\|_{\mathcal{E}^{v}(D)}}{v-1}=\frac{2 v-1}{v-1}\|u\|_{\mathcal{E}^{v}(D)} .
$$

It follows that $\left\{u \in \mathcal{E}^{\sqrt{v}}(D): \sup _{x \in \Omega} \rho^{l}(x)\left|D^{\alpha} u(x)\right|<\infty\right.$ for all $\alpha$ and $\left.l \in \mathbb{N}_{0}\right\} \subset \mathcal{E}^{v}(A)$. 
On the other hand, applying [8, Thm 6.5.2/1, Lemma 6.2.3] for any $k \in \mathbb{N}$, we obtain

$$
\begin{aligned}
\left\|A^{k} u\right\|_{L_{q}(\Omega)} & \geq c_{k}\|u\|_{W_{q}^{2 m k}(\Omega ; \rho q \mu k ; \rho q \tau k)} \\
& =c_{k}\left[\int_{\Omega}\left(\sum_{|\alpha|=2 m k} \rho^{q \mu k}(x)\left|D^{\alpha} u(x)\right|^{q}+\rho^{q \tau k}(x)|u(x)|^{q}\right) d x\right]^{\frac{1}{q}} \\
& \geq c_{k} c_{\rho}^{k}\left[\int_{\Omega}\left(\sum_{|\alpha|=2 m k}\left|D^{\alpha} u(x)\right|^{q}+|u(x)|^{q}\right) d x\right]^{\frac{1}{q}}=c_{k} c_{\rho}^{k}\|u\|_{W_{q}^{2 m k}(\Omega)^{\prime}}
\end{aligned}
$$

where $c_{\rho}>0$ does not depend on $k$. Thus,

$$
\begin{aligned}
\left\|A^{k+1} u\right\|_{L_{q}(\Omega)} & =\left\|A^{k}(A u)\right\|_{L_{q}(\Omega)} \geq c_{k} c_{\rho}^{k}\|A u\|_{W_{q}^{2 m k}(\Omega)} \\
& =c_{k} c_{\rho}^{k}\left(\sum_{|\alpha|=2 m k}\left\|D^{\alpha} A u\right\|_{L_{q}(\Omega)}^{q}+\|A u\|_{L_{q}(\Omega)}^{q}\right)^{\frac{1}{q}} \\
& \geq c_{k} c_{\rho}^{k}\left(\sum_{|\alpha|=2 m k}\left\|A D^{\alpha} u\right\|_{L_{q}(\Omega)}^{q}+\|A u\|_{L_{q}(\Omega)}^{q}\right)^{\frac{1}{q}} \\
& \geq c_{k} c_{1} c_{\rho}^{k+1}\left(\sum_{|\alpha|=2 m k}\left\|D^{\alpha} u\right\|_{W_{q}^{2 m}(\Omega)}^{q}+\|u\|_{W_{q}^{2 m}(\Omega)}^{q}\right)^{\frac{1}{q}}=c_{k+1} c_{\rho}^{k+1}\|u\|_{W_{q}^{2 m(k+1)}(\Omega)},
\end{aligned}
$$

where $c_{k+1}=c_{k} c_{1}=c_{1}^{k+1}$ by induction on $k$. Hence, for each $k \in \mathbb{N}$ and $u \in \mathfrak{D}^{k}(A)$, we have $\left\|A^{k} u\right\|_{L_{q}(\Omega)} \geq c_{1}^{k} c_{\rho}^{k}\|u\|_{W_{q}^{2 m k}(\Omega)}$ for all $u \in \mathfrak{D}^{k}(A)$, where $c_{1}>0$ does not depend on $k$. This leads to the inequality $\sum v^{-k}\left\|A^{k} u\right\|_{L_{q}(\Omega)} \geq \sum\left(\left(c_{1} c_{\rho}\right)^{-1} v\right)^{-k}\|u\|_{W_{q}^{k}(\Omega)}$ from which it follows that

$$
\mathcal{E}^{v}(A) \subset\left\{u \in \mathcal{E}^{\left(c_{1} c_{\rho}\right)^{-1} v}(D): \sup _{x \in \Omega} \rho^{l}(x)\left|D^{\alpha} u(x)\right|<\infty \text { for all } \alpha \text { and } l \in \mathbb{N}_{0}\right\} .
$$

Hence, equality (7) holds. Now applying [3, Thm 9], we obtain the required equality (6).

Using (5) and [4, Thm 2], as well as, taking into account (7), we obtain the required inequalities (2), (3), while (4) directly follows from (3) and [3, Thm 6].

\section{REFERENCES}

[1] Bergh J., Löfström J. Interpolation Spaces. Springer-Verlag, Berlin-Heidelberg-New York, 1976.

[2] Dmytryshyn M., Lopushansky O. Operator calculus on the exponential type vectors of operators with point spectrum. In book: Topology in Banach Spaces, 137-145. Nova, Huntigton, New York, 2001.

[3] Dmytryshyn M., Lopushansky O. Bernstein-Jackson-type inequalities and Besov spaces associated with unbounded operators. J. Inequal. Appl. 2014, 2014 (105), 1-12. doi:10.1186/1029-242X-2014-105

[4] Dmytryshyn M., Lopushansky O. On Spectral Approximations of Unbounded Operators. Complex Anal. Oper. Theory 2019, 1-15. doi:10.1007/s11785-019-00923-0

[5] Giulini S. Approximation and Besov spaces on stratified groups. Proc. Amer. Math. Soc. 1986, 96 (4), 569-578. doi:10.1090/S0002-9939-1986-0826483-3

[6] Gorbachuk M., Gorbachuk V. Approximation of smooth vectors of a closed operator by entire vectors of exponential type. Ukrainian Math. J. 1995, 47 (5), 713-726. doi:10.1007/BF01059045 
[7] Gorbachuk, M.L., Hrushka, Ya.I., Torba S.M. Direct and Inverse Theorems in the Theory of Approximation by the Ritz Method. Ukrainian Math. J. 2005, 57 (5), 751-764. doi:10.1007/s11253-005-0225-4

[8] Triebel H. Interpolation theory. Function spaces. Differential operators. North-Holland Publishing Company, Amsterdam-New York-Oxford, 1978.

Received 26.12.2018

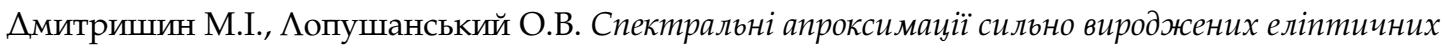
диферениіальних операторів // Карпатські матем. публ. - 2019. - Т.11, №1. - С. 48-53.

Встановлено аналітичні оцінки помилок спектральних апроксимацій сильно вироджених еліптичних диференціальних операторів в просторі $\Lambda$ ебега $L_{q}(\Omega)$ над обмеженою областю $\Omega$. Такі еліптичні оператори характеризуються сильним виродженням їх коефіцієнтів поблизу границі, їх спектр складається із ізольованих власних значень скінченної алгебраїчної кратності, а лінійна оболонка власних і приєднаних векторів щільна в просторі $L_{q}(\Omega)$. Отримані

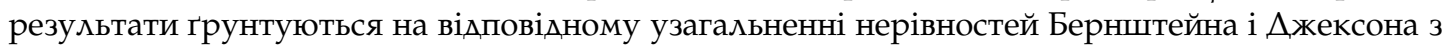
обчисленням точних констант для квазінормованих апроксимаційних просторів типу Бєсова, асоційованих з даним еліптичним оператором. Апроксимаційні простори визначаються за допомогою функціоналу $E(t, u)$, який характеризує найкоротшу відстань від заданої функції $u \in L_{q}(\Omega)$ до замкненої лінійної оболонки спектральних підпросторів заданого оператора, шо відповідають власним значенням, які за абсолютною величиною не перевишують фіксоване число $t>0$. При цьому вказана лінійна оболонка спектральних підпросторів співпадає з пілпростором цілих аналітичних функцій експоненціального типу, що не перевищує $t>0$. Апроксимаційний функціонал $E(t, u)$ в нашому випадку відіграє роль, подібну модулю гладкості в теорії функцій.

Ключові слова і фрази: еліптичні оператори, спектральні апроксимації. 